\title{
Comparison of Automated Anti-Müllerian Hormone Assays and Antral Follicle Count in Predicting Ovarian Response During Ovarian Stimulation
}

\author{
Eek Chaw Tan ${ }^{1}$, Pallavi Chincholkar ${ }^{2}$, Su Ling Yu ${ }^{1,3,4}$, Serene Liqing Lim ${ }^{1,4}$, Rajkumaralal Renuka ${ }^{1}$, \\ Tze Tein Yong ${ }^{1,3,4}$, Chin Pin Yeo ${ }^{2}$, Hemashree Rajesh ${ }^{1,3,4 *}$ \\ ${ }^{1}$ Department of Obstetrics \& Gynaecology, Centre for Assisted Reproduction, Singapore General Hospital, Singapore \\ ${ }^{2}$ Department of Clinical Pathology, Singapore General Hospital, 20 College Road, Singapore 169856 \\ ${ }^{3}$ Duke-NUS Medical School, 8 College Rd, Singapore 169857 \\ ${ }^{4}$ Yong Loo Lin School of Medicine, NUHS Tower Block, 1E Kent Ridge Road, Singapore 119228
}

\begin{abstract}
Objective: Various parameters had been used to predict ovarian response. Among them, Anti-Müllerian Hormone (AMH) and antral follicle count (AFC) demonstrate the most favourable analytical and performance characteristics. In this pilot study, we aim to determine the cut-off levels of AMH using automated AMH assays and AFC in the prediction of poor and high responders.

Study Design: Prospective study of 43 women between 21 to 45 years old scheduled for assisted reproduction. AMH levels on day 3 of menstruation were analysed using two immunoassay kits, namely the Beckman Coulter Access AMH and the Roche Elecsys AMH on the two automated analysers Beckman Coulter DxI 800 and Roche Cobas e602 respectively. AFC was also assessed on day 3 of menstruation prior to in vitro fertilization (IVF). These were compared with the number of oocytes retrieved after controlled ovarian stimulation.

Results: AMH (Beckman Coulter Access AMH and Roche Elecsys AMH) highly correlated with AFC and the number of oocytes retrieved after ovarian stimulation. Beckman Coulter Access AMH was the better predictor for poor ovarian response with $\mathrm{ROC}_{\mathrm{AUC}}$ of 0.83 . For the prediction of a high response, AFC had a higher ROC ${ }_{\text {AUC }}$ of 0.95 . Through ROC, the AMH cut-off level for poor ovarian response was $2.23 \mathrm{ng} / \mathrm{ml}$ with Beckman Coulter Access AMH and $2.02 \mathrm{ng} / \mathrm{ml}$ with Roche Elecsys AMH, while the AMH cut-off for a high ovarian response was $5.19 \mathrm{ng} / \mathrm{ml}$ with Beckman Coulter Access AMH and $4.60 \mathrm{ng} / \mathrm{ml}$ with Roche Elecsys $\mathrm{AMH}$. For AFC, the cut-off for poor ovarian response was 18 and for high response was 34.

Conclusion: AMH and AFC are reliable predictors of ovarian response. Establishment of specific levels may improve individualised controlled ovarian stimulation and optimise the oocyte yield. Larger studies are required to establish these findings.
\end{abstract}

Keywords: Anti-Müllerian Hormone; Antral Follicle Count.

\section{INTRODUCTION}

Anti-Müllerian Hormone (AMH) is a dimeric glycoprotein member of the transforming growth factor-beta superfamily (Cate et al., 1986; Jost, 1946). It is expressed in granulosa cells from pre-antral and small antral follicles (Durlinger et al., 2002a, 2002b) and continues to be expressed in the growing follicles in the ovary until they have reached the size and differentiation state at which they are to be selected for dominance. In women, AMH appears in serum after birth, increases till puberty and progressively decreases in parallel with ovarian ageing (de Vet et al., 2002; van Rooij et al., 2005). This is because in women AMH is produced by granulosa cells, from preantral and antral follicles (Weenen et al., 2004) and its levels reflect the ovarian follicular pool. Therefore, AMH serum levels can be used as a marker for ovarian reserve, representing the quantity and quality of the ovarian follicle pool (Te Velde and Pearson, 2002).

$\mathrm{FSH}$, inhibin B and antral follicle count (AFC) have been used to measure ovarian reserve and predict ovarian response in In Vitro Fertilization (IVF) patients for decades. However, they fluctuate throughout the menstrual cycle and need to be measured in the early follicular phase of the menstrual cycle for more accuracy. In contrast, there is little intra-cycle variation in AMH levels, supporting feasibility of assessment throughout the cycle (Cook et al., 2000). Furthermore, studies have shown that AMH levels remain unchanged in response to gonadotrophin stimulation (van Rooij et al., 2002).

Many studies have been done to assess the sensitivity of AMH in predicting ovarian reserve and response in IVF patients, especially in

() 2019 by the Asia Pacific Initiative on Reproduction (ASPIRE) and World Scientific Publishing Co. Pte. Ltd. $\odot$ Open Access article under the CC BY-NC-ND license (http://creativecommons.org/licenses/by-nc-nd/4.0/).

Received 24 January 2019; Accepted 12 May 2019; Published 17 June 2019

${ }^{*}$ Correspondence should be addressed to: Dr. Hemashree Rajesh, Department of Obstetrics \& Gynaecology, Centre for Assisted Reproduction, Singapore General Hospital, Outram Road, Block 5, Level 1, Singapore 169608. Email: hemashreerajesh@yahoo.com 
western countries. However, it is unknown whether AMH levels vary between populations. We prospectively assessed the significance of $\mathrm{AMH}$ as a marker of ovarian reserve and compared with other parameters (antral follicle count, FSH, E2 and age) to predict the number of oocytes retrieved during IVF in the Singapore population.

\section{SUBJECTS AND METHODS}

The study was conducted at the Singapore General Hospital, Centre for Assisted Reproduction. Approval was obtained from the local institutional review board.

Patients scheduled to undergo their first stimulated cycle were recruited. Written informed consent was obtained from all participants.

The inclusion criteria were as follows: 1) age between 21 to 45 years, 2) regular menstrual cycles with cycle length less than or equal to 35 days, 3) $\mathrm{BMI}<25,4$ ) never pregnant or at least 6 weeks from the most recent pregnancy.

The exclusion criteria were as follows: 1) age above 45 or below 21 years old, 2) irregular menstrual cycles with cycle length more than 35 days, 3) current or previous diagnosis of PCOS, 4) current or previous diagnosis of endocrine or metabolic conditions e.g. thyroid disorder, 5) current or prior use of hormonal contraception, 6) postmenopausal status, 7) current or previous chemotherapy recipient, 8) current or previous diagnosis of malignancy, 9) current or ex-smoker, 10) alcohol consumption more than 3 standard drinks per day (a standard drink is defined as one 12-ounce bottle of beer, one 5-ounce glass of wine, or 1.5 ounces of distilled spirits), 11) FSH levels more than $10 \mathrm{U} / \mathrm{L}$ on day 3 of menstrual cycle. On day 3 of a spontaneous menstrual cycle, patients underwent a transvaginal ultrasound examination using a GE Voluson E8 ultrasound system (GE Healthcare, Milwaukee, WI, USA) equipped with a 6-12 $\mathrm{MHz} 3 \mathrm{D}$ endovaginal probe to assess the number of antral follicles measuring 2-10 $\mathrm{mm}$. The scans were conducted by 4 professional sonographers who rotate to our centre every 3 months. Venous blood samples were also obtained on day 3 of menstruation for the measurement of $\mathrm{AMH}$, follicular stimulating hormone (FSH) and estradiol (E2) levels. Samples were centrifuged at $3500 \mathrm{rpm}$ for 5 minutes and serum stored at $-20^{\circ} \mathrm{C}$ until assayed for up to 1 month.

The Beckman Coulter Access AMH and Roche Elecsys AMH immunoassays were compared. Beckman Coulter Access AMH assay is traceable to internal reference standards which consist of recombinant AMH in a BSA matrix. Roche Elecsys AMH assay is traceable to the Beckman Coulter AMH Gen II ELISA (unmodified version without predilution) assay. In Table 1, the assay characteristics of both Beckman Coulter Access AMH and Roche Elecsys AMH are shown. For Beckman Coulter Access AMH, manufacturer's imprecision claim was $\leq 0.032 \mathrm{ng} / \mathrm{mL}$ (SD) at AMH $<0.16 \mathrm{ng} / \mathrm{mL}$ and $\leq 10.0 \%(\mathrm{CV})$ at concentrations $\geq 0.16 \mathrm{ng} / \mathrm{mL}$. For Roche Elecsys AMH the manufacturer's claim for total imprecision was $<0.03 \mathrm{ng} / \mathrm{mL}(\mathrm{SD})$ at $0.01-0.5 \mathrm{ng} / \mathrm{mL}, \leq 6 \%(\mathrm{CV})$ at $\mathrm{AMH}$ $\geq 0.5-1 \mathrm{ng} / \mathrm{mL}$ and $\leq 5 \%(\mathrm{CV})$ at $\mathrm{AMH} \geq 1 \mathrm{ng} / \mathrm{mL}$ respectively. FSH and E2 were assessed using the Roche Elecsys FSH \& E2 assay kits on the Roche Cobas e602 analyser.

All patients had an antagonist cycle. Patient with an AFC of $<14$ were given a dose of $450 \mathrm{IU}$ of follitropin alpha or beta. Patients with follicles between 14-24 received 150-300 IU of follitropin alpha or beta based on their BMI. Patients with an AFC $>24$ received 150 IU of follitropin alpha or beta. If there were $>19$ follicles $>11 \mathrm{~mm}$

Table 1. AMH assay characteristics.

\begin{tabular}{lll}
\hline AMH assays & \multicolumn{1}{c}{ Beckman Coulter Access AMH } & \multicolumn{1}{c}{ Roche Elecsys AMH } \\
\hline Total imprecision & $\leq 0.032 \mathrm{ng} / \mathrm{mL}(\mathrm{SD})$ at $<0.16 \mathrm{ng} / \mathrm{mL}$ & $<0.03 \mathrm{ng} / \mathrm{mL}(\mathrm{SD})$ at $0.01-0.5 \mathrm{ng} / \mathrm{mL}$ \\
& $\leq 10.0 \%(\mathrm{CV})$ at $\geq 0.16 \mathrm{ng} / \mathrm{mL}$ & $\leq 6 \%(\mathrm{CV})$ at $\geq 0.5-1 \mathrm{ng} / \mathrm{mL}$ \\
& Serum & $\leq 5 \%(\mathrm{CV})$ at $\geq 1 \mathrm{ng} / \mathrm{mL}$ \\
Sample type & $20 \mu \mathrm{l}$ & Serum \\
Minimum sample volume & $39 \mathrm{~min}$ & $50 \mu \mathrm{l}$ \\
Incubation time & $\leq 0.02 \mathrm{ng} / \mathrm{mL}$ & $18 \mathrm{~min}$ \\
Limit of Detection (LoD) & $\leq 0.08 \mathrm{ng} / \mathrm{mL}$ & $0.01 \mathrm{ng} / \mathrm{mL}$ \\
Limit of Quantification (LoQ) & $0.02-24 \mathrm{ng} / \mathrm{mL}$ & $0.03 \mathrm{ng} / \mathrm{mL}$ \\
Analytical measurement range & & $0.01-23 \mathrm{ng} / \mathrm{mL}$ \\
\hline
\end{tabular}

Table 2. Patient characteristics (mean of age, AMH, AFC, FSH, E2 and number of oocytes retrieved).

\begin{tabular}{|c|c|c|c|c|c|c|}
\hline & Total $(n=43)$ & Poor responder $(n=11)$ & Normal $(n=26)$ & High responder $(n=6)$ & $P$ value & Standard deviation \\
\hline Age (years) & 33.6 & 35.1 & 33.4 & 32.0 & 0.30 & 4.08 \\
\hline Beckman Coulter Access AMH level (ng/mL) & 3.80 & 1.59 & 3.51 & 9.12 & $<0.001$ & 3.44 \\
\hline Roche Elecsys AMH level (ng/mL) & 3.17 & 1.43 & 2.88 & 7.61 & $<0.001$ & 2.79 \\
\hline $\operatorname{AFC}(n)$ & 22.86 & 13.09 & 21.73 & 45.67 & $<0.001$ & 16.17 \\
\hline Roche Elecsys FSH (IU/L) & 6.77 & 8.76 & 6.17 & 5.72 & 0.119 & 3.75 \\
\hline Roche Elecsys E2 (pmol/L) & 195.06 & 257.31 & 181.30 & 123.26 & 0.240 & 162.19 \\
\hline Number of oocytes (n) & 10.02 & 2.55 & 10.19 & 23.00 & NA & 7.00 \\
\hline
\end{tabular}


on trigger day, a lucrin $1 \mathrm{mg}$ trigger was administered. Otherwise all patients received a standard dose of 10,000 IU of HCG as trigger.

When three or more follicles reached $>18 \mathrm{~mm}$, HCG or GnRH analogue was administered and follicles were aspirated 36 hours later under sedation. The main outcome measures were the number of oocytes retrieved and the cycle cancellation rate. Poor ovarian response was defined as a fewer than five oocytes or cancellation due to impaired or absent follicular growth in response to ovarian stimulation. (Bancsi et al., 2002). A normal ovarian response was defined as a collection of 5-16 oocytes. Patients were considered high responders when $>16$ oocytes were collected at ovum retrieval or when the cycle was cancelled because of exaggerated response (Muttukrishna et al., 2004).

Data were analysed with the Statistical Program for Social Sciences version 22 (SPSS Inc., Chicago, IL, USA). Values are presented as mean. To compare poor, normal and high responders the

Fig. 1. Correlation of age (A), cycle day 3 E2 (B), number of oocytes after oocyte retrieval (C), cycle day 3 number of antral follicles (D) and cycle day 3 FSH (E) with Beckman Coulter Access AMH in IVF patients.

Spearman's correlation coefficient is " $r$ " followed by the "P" value.
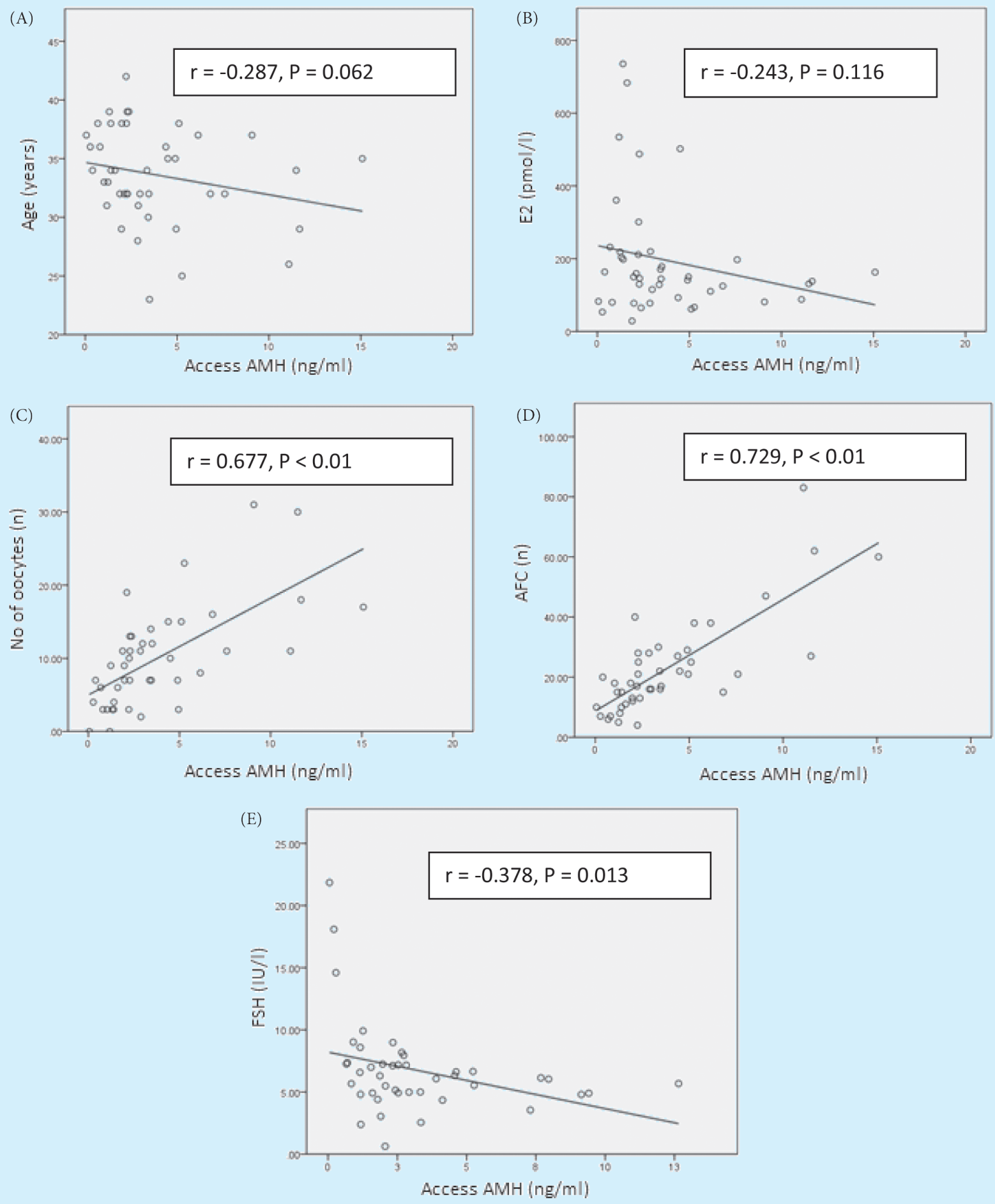
ANOVA test was performed whenever appropriate. The correlation between different parameters is expressed as Spearman's correlation coefficient. All variables were considered significant at a $\mathrm{P}$ value of less than 0.05. The AMH and AFC cut-offs for poor and high ovarian response of $\mathrm{AMH}$ and $\mathrm{AFC}$ were derived from $\mathrm{ROC}_{\mathrm{AUC}}$.

\section{RESULTS}

Forty-three patients who underwent their first stimulated IVF cycle were included in the analysis.

Patient and ovarian reserve test characteristics of poor, normal and high responders are compared in Table 2. AMH levels and AFC measured were significantly lower in the poor responder group compared to the normal and high responders. The numbers of oocytes retrieved in the poor, normal and high responders are also depicted in Table 2. Two patients in the poor responders group had their oocyte retrieval cancelled because of insufficient follicle growth.

The correlation coefficients for the association between automated AMH levels (Beckman Coulter Access and Roche Elecsys) on one hand and several reserve test variables and the total numbers of oocytes retrieved on the other hand are shown in Figs. 1 and 2. AMH (Beckman Coulter Access and Roche Elecsys) were highly correlated with AFC and the number of oocytes retrieved after ovarian stimulation.

In Table 3, the results of the logistic regression analysis for the prediction of poor response are given. Beckman Coulter Access $\mathrm{AMH}$ presented the highest $\mathrm{ROC}{ }_{\mathrm{AUC}}$ of 0.83 , indicating a good discriminating potential for predicting poor ovarian response. The ROC $_{\text {AUC }}$ for Roche Elecsys AMH was almost identical, followed by the ROC ${ }_{\text {AUC }}$ value of AFC. Age, FSH and E2 were not significantly related to poor response.

In Table 4, the results of the logistic regression analysis for the prediction of high response are given. AFC presented the highest ROC ${ }_{\text {AUC }}$ of 0.95 , indicating a good discrimination potential for predicting high ovarian response followed by the $\mathrm{ROC}_{\mathrm{AUC}}$ value of AMH (Beckman Coulter Access and Roche Elecsys). Age, FSH and E2 were not significantly related to high response.

Through ROC, it can be derived that the AMH cut-off level for poor ovarian response are $2.23 \mathrm{ng} / \mathrm{ml}$ in Beckman Coulter Access AMH with the sensitivity of $81.8 \%$ and specificity of $75.0 \%$ and $2.02 \mathrm{ng} /$ $\mathrm{ml}$ in Roche Elecsys AMH with the sensitivity of $81.8 \%$ and specificity of $71.9 \%$. While the AMH cut-off level for high ovarian response are $5.19 \mathrm{ng} / \mathrm{ml}$ in Beckman Coulter Access AMH with the sensitivity of $83 \%$ and specificity of $89.2 \%$ and $4.60 \mathrm{ng} / \mathrm{ml}$ in Roche Elecsys AMH with the sensitivity of $83 \%$ and specificity of $98.2 \%$. For antral follicle count, the cut-off for poor ovarian response is 17.5 (sensitivity of $81.8 \%$ and specificity of $65.6 \%$ ) whereas the cut-off for high ovarian response is 34 (sensitivity of $83.3 \%$ and specificity of $94.6 \%$ ).

\section{DISCUSSION}

In this study we prospectively compared the prediction rate of $\mathrm{AMH}$, $\mathrm{AFC}, \mathrm{FSH}, \mathrm{E} 2$ and age in assessing ovarian response of patients undergoing their first IVF cycle. We evaluated the number of oocytes retrieved during first IVF to evaluate the predictive comparison of $\mathrm{AMH}$ and AFC for the identification of a poor or a high responder. Several studies had proposed that among the wide variety of biomarkers used for the prediction of ovarian response $\mathrm{AMH}$ and AFC demonstrated the highest reliability (Broekmans et al., 2006; Broer et al., 2009, 2011).

Studies using manual enzyme-linked immunosorbent assays have reported the sensitivity and specificity of $\mathrm{AMH}$ to range from $44-97 \%$ and $41-100 \%$ respectively in predicting poor ovarian response. This was partly attributed to the use of different variants of AMH assay with variable cut-offs depending on the assay used (La Marca et al., 2010). In this study, we compared the performance of two automated immunoassays (Beckman Coulter Access AMH and Roche Elecsys AMH). Roche Elecsys AMH assay on the Cobas e602 analyser demonstrated a lower imprecision and lower limit of detection when compared to Beckman Coulter AMH on the Beckman Coulter DxI800 analyser (Table 1). Furthermore, our study showed that mean of AMH values obtained from the Beckman Coulter Access AMH are 1.27\% higher than Roche Elecsys AMH. Teddy et al. had demonstrated that the Beckman Coulter Access AMH were 5\% higher than those obtained with Roche Elecsys AMH (Tadros et al., 2016). It could be due to different antigens are used in both assays. Nonetheless, the excellent correlation between the two assays demonstrated by our study will still allow comparison of results obtained using both assays. In our study, Beckman Coulter Access AMH showed good discriminating potential for the prediction of poor ovarian response with highest ROC $_{\text {AUC }}$ of 0.83 .

Previous study reported that AMH is cycle independent in the assessment of ovarian response as it had less intra- and inter-cycle fluctuation (Iliodromiti et al., 2015; van Disseldorp et al., 2010). Andersen et al. found that $\mathrm{AMH}$ was a significant predictive variable in the assessment of poor and high ovarian response during ovarian stimulation whereas no such independent association was observed with AFC (Andersen et al., 2011). AMH levels do not change in response to an acute endogenous rise in FSH and LH (Durlinger et al., 1999; van Rooij et al., 2002).

However, recent studies have revealed important inter and intracycle variations during ovarian stimulation for ART that cannot be explained only by analytical variability. Serum AMH levels are lower in the luteal phase than the follicular phase (Gorkem et al., 2017). The average intraindividual $\mathrm{AMH}$ variability in a menstrual cycle was $20 \%$ and this biological variation was at least twice the analytical variation (Hadlow et al., 2016). Furthermore, intracycle variation in younger women were different from older women (Overbeek et al., 2012). Short-term inter-cycle variation may be due to the biological variation in the number of AMH-producing follicles in a cycle (Rombauts et al., 2015).

During ovarian stimulation, serum AMH levels gradually decrease in follicular phase irrespective to the type of FSH used or the protocol (Fanchin et al., 2003; Lee et al., 2010; Melado Vidales et al., 2017a; Weintraub et al., 2014). This decline continues till upto 4 days after HCG administration. Then, AMH increases and returns to baseline levels regardless of whether the patient is pregnant of not (Fanchin et al., 2005; Fanchin et al., 2003; Weintraub et al., 2014).

Basal AMH levels on days 1-3 of cycle has shown a good correlation with the number of oocytes retrieved (Lee et al., 2010; Melado Vidales et al., 2017b). Until the dynamics of AMH are further clarified, standardization of measuring $\mathrm{AMH}$ during the early follicular phase will provide the most accurate result for hormone stimulation during IVF.

The advantages of AMH is that it is not produced by atretic antral follicles which do not respond to ovarian stimulation which cannot be excluded by an AFC measurement. AMH however has a disadvantage in that, it may overestimate the ovarian response of patients in whom the majority of follicles are 1-2 $\mathrm{mm}$ compared to those who have more large antral follicles $(>6 \mathrm{~mm})$. Since the large follicles are most sensitive to gonadotropin treatment while simultaneously losing their ability to produce AMH, AFC might be a better predictor of the immediate ovarian response to gonadotropin stimulation than AMH (Mutlu et al., 2013).

In general, the performance characteristics of AFC in predicting the number of oocytes retrieved is comparable to that of $\mathrm{AMH}$. 
Fig. 2. Correlation of age (A), cycle day 3 E2 (B), number of oocytes after oocyte retrieval (C), cycle day 3 number of antral follicles (D) and cycle day 3 FSH (E) with Roche Elecsys AMH in IVF patients.

Spearman's correlation coefficient is " $r$ " followed by the " $\mathrm{P}$ " value.

(A)
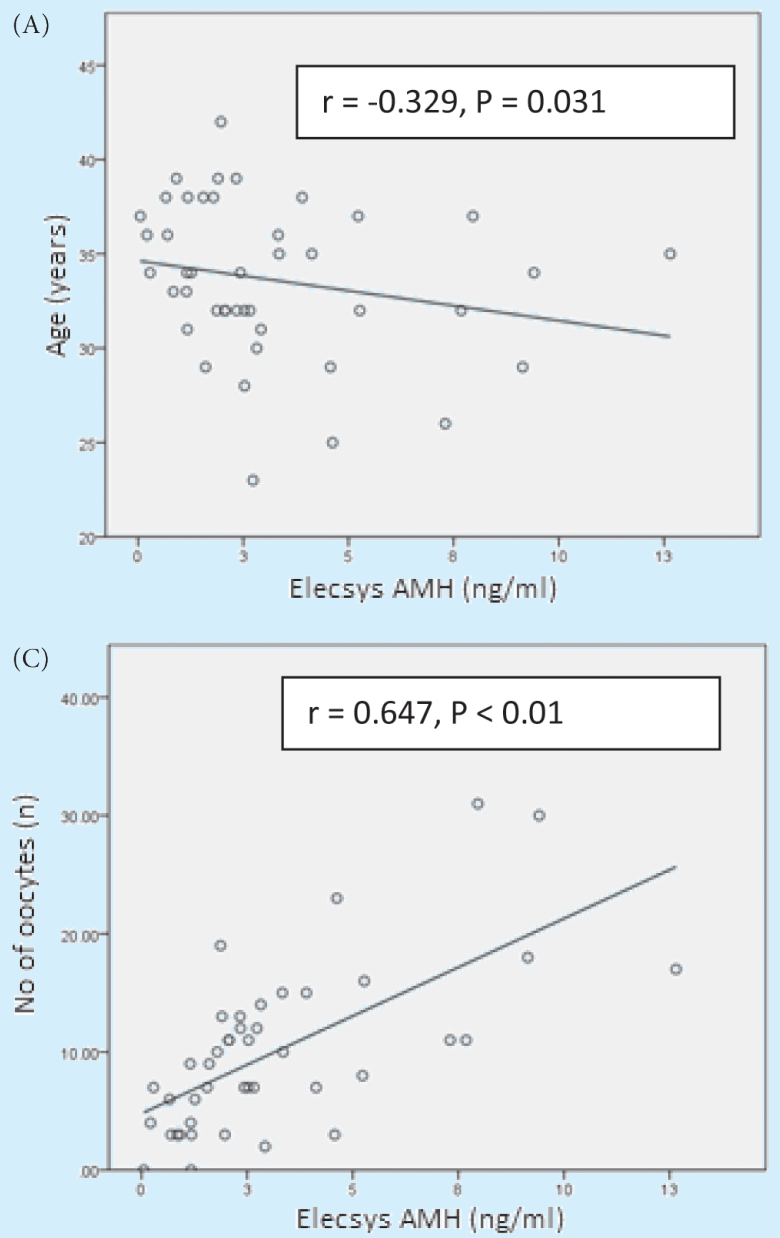

(B)
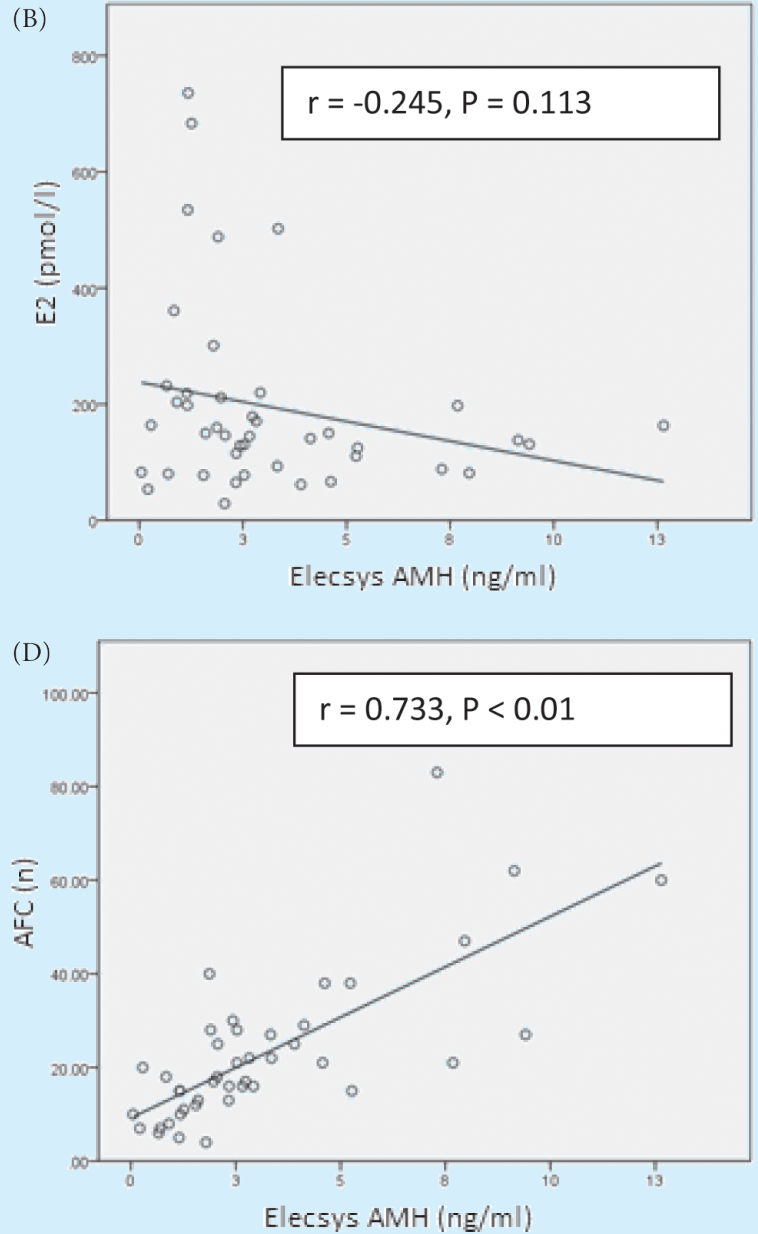

(E)

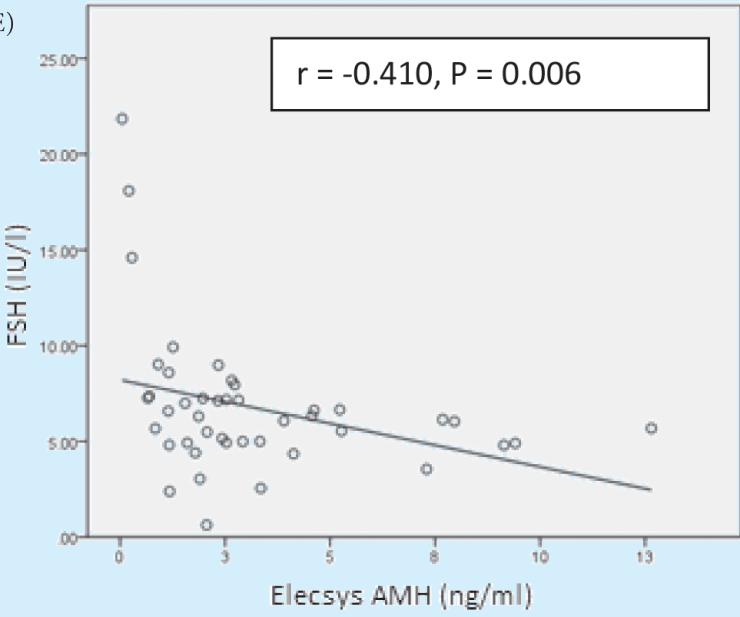

Van Rooij et al. had showed that AFC is a slightly better predictor for poor ovarian response than AMH (manual immunoassay) with ROC $_{\text {AUC }}$ of 0.86 compared to 0.85 in AMH (van Rooij et al., 2002). In our study, $\mathrm{AMH}$ showed a higher $\mathrm{ROC}_{\mathrm{AUC}}$ than $\mathrm{AFC}$ in predicting poor ovarian response. However, our study also demonstrated that $\mathrm{AFC}$ is a better marker for predicting high ovarian response with a $\mathrm{ROC}_{\mathrm{AUC}}$ of 0.95 compared to 0.88 for $\mathrm{AMH}$.
One advantage of AFC is that the results are immediate whereas AMH may be associated with a turnaround time that is dependent on the local laboratory's workflow. In addition, the observer is able to evaluate many other important aspects of the ovaries, such as their position and accessibility, the presence of endometrioma or other ovarian lesions, concomitant tubal pathology, and uterine abnormalities (endometrial polyps, adenomyosis or leiomyoma). 
Table 3. Logistic regression for prediction of poor response following ovarian hyperstimulation.

\begin{tabular}{lccc}
\hline Univariate analysis & Odds ratio $(95 \% \mathrm{Cl})$ & P value & ROC $_{\text {AUC }}$ \\
\hline AFC (per follicle) & $0.88(0.79-0.98)$ & 0.021 & 0.79 \\
Beckman Access AMH (per ng/mL) & $0.45(0.23-0.45)$ & 0.022 & 0.83 \\
Roche Elecsys AMH (per ng/mL) & $0.43(0.20-0.91)$ & 0.026 & 0.81 \\
Roche Elecsys FSH (per IU/L) & $1.20(0.98-1.47)$ & 0.076 & 0.65 \\
Roche Elecsys E2 (per pmol/L) & $1.00(1.00-1.01)$ & 0.156 & 0.66 \\
Age (per year) & $1.14(0.94-1.39)$ & 0.173 & 0.61 \\
\hline
\end{tabular}

Table 4. Logistic regression for prediction of high response following ovarian hyperstimulation.

\begin{tabular}{lccc}
\hline Univariate analysis & Odds ratio $(\mathbf{9 5} \% \mathrm{Cl})$ & P value & ROC $_{\text {AUC }}$ \\
\hline AFC (per follicle) & $1.10(1.02-1.17)$ & 0.008 & 0.95 \\
Beckman Coulter Access AMH (per ng/mL) & $1.62(1.17-2.23)$ & 0.003 & 0.88 \\
Roche Elecsys AMH (per ng/mL) & $1.91(1.24-2.94)$ & 0.003 & 0.88 \\
Roche Elecsys FSH (per IU/L) & $0.88(0.63-1.24)$ & 0.461 & 0.62 \\
Roche Elecsys E2 (per pmol/L) & $0.99(0.98-1.01)$ & 0.275 & 0.64 \\
Age (per year) & $0.89(0.73-1.10)$ & 0.295 & 0.62 \\
\hline
\end{tabular}

The key limitation of AFC however is the lack of standardization for measurement which may underlie its limited transportability across different operators, sites and settings (Iliodromiti et al., 2015). Unlike AMH, AFC exhibits significant variation within and across consecutive cycles (Deb et al., 2013; Elter et al., 2005; Hansen et al., 2003; van Disseldorp et al., 2010). Assessment of the intra-class coefficient (ICC) of AFC showed that it had a modest ICC of 0.71 between two cycles and of 0.69 within one cycle. This was substantially worse than that for AMH which showed an ICC between two cycles and within one cycle of 0.89 and 0.87 respectively (van Disseldorp et al., 2010). Given these concerns a consensus workgroup suggested that AFC should be performed from day 2 to day 4 of an index cycle (Broekmans et al., 2010).

\section{STRENGTH AND LIMITATIONS}

This is a prospective study on determining $\mathrm{AMH}$ cut-off levels using automated AMH assays and AFC in the prediction of poor and high responders in a Singapore population. The results of this study can be used to guide clinical practice.

The limitation of this study was the small sample size. Hence, further studies with larger sample sizes are needed to prove statistical significance. Furthermore, the AFC scan was conducted by professional sonographers (not MD holders) in our centre with a high-resolution ultrasound machine (GE Voluson E8 ultrasound system). The high resolution of the machine and the experience of our sonographer may explain why our cut-off for AFC for the poor and high responders are relatively higher compared to other studies. Besides, different dosing of gonadotropin was used based on the follicular count and BMI. These confounding factors may have affected the outcomes.

However, there have not been any publications corelating both $\mathrm{AFC}$ and $\mathrm{AMH}$ to oocyte number. Hence, this novel concept was put forth. Further studies involving a larger number of patients with strict protocols may establish more robust values.

\section{CONCLUSION}

$\mathrm{AMH}$ and AFC are good predictive markers for ovarian response. Establishment of specific levels may improve individualised controlled ovarian stimulation and optimize oocyte recovery. Larger studies with more patients and strict stimulation protocol can give us a better conclusion of AMH and AFC predictability of oocyte number. This study suggested that both the Beckman Coulter Access $\mathrm{AMH}$ and Roche Elecsys AMH assays are concordant with each other and reliably related to the number of oocytes retrieved during IVF. However, clinicians should be aware of variations in absolute values between both assays.

\section{ACKNOWLEDGEMENTS}

Beckman Coulter and Roche Diagnostics provided assay kits for the study.

\section{REFERENCES}

Andersen AN, Witjes H, Gordon K, Mannaerts B. Predictive factors of ovarian response and clinical outcome after IVF/ICSI following a rFSH/GnRH antagonist protocol with or without oral contraceptive pre-treatment. Hum Reprod. 2011;26: 3413-23.

Bancsi LF, Broekmans FJ, Eijkemans MJ, de Jong FH, Habbema JD, te Velde ER. Predictors of poor ovarian response in in vitro fertilization: a prospective study comparing basal markers of ovarian reserve. Fertil Steril. 2002;77:328-36.

Broekmans FJ, de Ziegler D, Howles CM, Gougeon A, Trew G, Olivennes F. The antral follicle count: practical recommendations for better standardization. Fertil Steril. 2010;94:1044-51.

Broekmans FJ, Kwee J, Hendriks DJ, Mol BW, Lambalk CB. A systematic review of tests predicting ovarian reserve and IVF outcome. Hum Reprod Update. 2006;12:685-718. 
Broer SL, Dolleman M, Opmeer BC, Fauser BC, Mol BW, Broekmans FJM. AMH and AFC as predictors of excessive response in controlled ovarian hyper stimulation: a meta-analysis. Hum Reprod Update. 2011;17:46-54.

Broer SL, Mol BWJ, Hendriks D, Broekmans FJM. The role of antiMüllerian hormone in prediction of outcome after IVF: comparison with the antral follicle count. Fertil Steril. 2009;91: 705-14.

Cate RL, Mattaliano RJ, Hession C, Tizard R, Farber NM, Cheung A, Ninfa EG, Frey AZ, Gash DJ, Chow EP. Isolation of the bovine and human genes for Müllerian inhibiting substance and expression of the human gene in animal cells. Cell. 1986;45:685-98.

Cook CL, Siow Y, Taylor S, Fallat ME. Serum Müllerian-inhibiting substance levels during normal menstrual cycles. Fertil Steril. 2000;73:859-61.

de Vet A, Laven JS, de Jong FH, Themmen AP, Fauser BC. AntiMüllerian hormone serum levels: a putative marker for ovarian aging. Fertil Steril. 2002;77:357-62.

Deb S, Campbell BK, Clewes JS, Pincott-Allen C, Raine-Fenning NJ. Intracycle variation in number of antral follicles stratified by size and in endocrine markers of ovarian reserve in women with normal ovulatory menstrual cycles. Ultrasound Obstet Gynecol. 2013;41:216-22.

Durlinger AL, Gruijters MJ, Kramer P, Karels B, Ingraham HA, Nachtigal MW, Uilenbroek JT, Grootegoed JA, Themmen AP. Anti-Müllerian hormone inhibits initiation of primordial follicle growth in the mouse ovary. Endocrinology. 2002a;143:1076-84.

Durlinger AL, Kramer P, Karels B, de Jong FH, Uilenbroek JT, Grootegoed A, Themmen AP. Control of primordial follicle recruitment by anti-Müllerian hormone in the mouse ovary. Endocrinology. 1999;140:5789-96.

Durlinger AL, Visser JA, Themmen AP. Regulation of ovarian function: the role of anti-Müllerian hormone. Reproduction. 2002b;124: 601-9.

Elter K, Sismanoglu A, Durmusoglu F. Intercycle variabilities of basal antral follicle count and ovarian volume in subfertile women and their relationship to reproductive aging: a prospective study. Gynecol Endocrinol. 2005;20:137-43.

Fanchin R, Méndez Lozano DH, Louafi N, et al. Dynamics of serum anti-Müllerian hormone levels during the luteal phase of controlled ovarian hyper stimulation. Hum Reprod. 2005;20(3):747-51.

Fanchin R, Schonauer LM, Righini C, et al. Serum anti-Müllerian hormone dynamics during controlled ovarian hyperstimulation. Hum Reprod. 2003;18:328-33.

Gorkem U, Kucukler FK, Togrul C, et al. Anti-Müllerian hormone exhibits a great variation in infertile women with different ovarian reserve patterns. Aust N Z J Obstet Gynaecol. 2017;57(4):464-8.

Hadlow N, Brown SJ, Habib A, et al. Quantifying the intraindividual variation of anti-Müllerian hormone in the ovarian cycle. Fertil Steril. 2016;106(5):1230-7.

Hansen KR, Morris JL, Thyer AC, Soules MR. Reproductive aging and variability in the ovarian antral follicle count: application in the clinical setting. Fertil Steril. 2003;80:577-83.

Jost A. Recherches sur la differenciation sexuelle de l'embryon de lapin. Arch Anat Microsc Morphol Exp. 1946;36:271-315.

La Marca A, Sighinolfi G, Radi D, Argento C, Baraldi E, Artenisio AC, Stabile G, Volpe A. Anti-Müllerian hormone (AMH) as a predictive marker in assisted reproductive technology (ART). Hum Reprod Update. 2010;16:113-30.
Lee JR, Kim SH, Kim SM, et al. Anti-Müllerian hormone dynamics during controlled ovarian hyper stimulations and optimal timing of measurement for outcome prediction. Hum Reprod. 2010; 25:2597-604.

lliodromiti S, Anderson RA, Nelson SM. Technical and performance characteristics of anti-Müllerian hormone and antral follicle count as biomarkers of ovarian response. Hum Reprod Update. 2015;21(6):698-710.

Melado Vidales L, Fernández-Nistal A, Martínez Fernández V, et al. Anti-Müllerian hormone dynamics during GNRH-antagonist short protocol for IVF/ICSI in women with varying ovarian reserve levels. Minerva Ginecol. 2017a;69(2):128-34.

Melado Vidales L, Fernández-Nistal A, Martínez Fernández V, et al. Anti-Müllerian hormone levels to predict oocyte maturity and embryo quality during controlled ovarian hyper stimulation. Minerva Ginecol. 2017b;69(3):225-32.

Mutlu MF, Erdem M, Erdem A, Yildiz S, Mutlu I, Arisoy O, Oktem M. Antral follicle count determines poor ovarian response better than anti-Müllerian hormone but age is the only predictor for live birth in in vitro fertilization cycles. J Assist Reprod Genet. 2013;30:657-65.

Muttukrishna S, Suharjono H, McGarrigle H, Sathanandan M. Inhibin $\mathrm{B}$ and anti-Müllerian hormone: markers of ovarian response in IVF/ICSI patients? BJOG. 2004;111:1248-53.

Overbeek A, Broekmans FJ, Hehenkamp WJ. Intra-cycle fluctuations of anti-Müllerian hormone in normal women with regular cycle: a reanalysis. Reprod Biomed Online. 2012;24:664-9.

Rombauts L, Lambalk CB, Schultze-Mosgau A, et al. Intercycle variability of the ovarian response in patients undergoing repeated stimulation with corifollitropin alfa in a gonadotropin-releasing hormone antagonist protocol. Fertil Steril. 2015;104(4):884-90.

Tadros T, Tarasconi B, Nassar J, Benhaim J-L, Taieb J, Fanchin R. New automated Anti-Müllerian hormone assays are more reliable than the manual assay in patients with reduced antral follicle count. Reprod Endocrinol. 2016;106:1800-6.

Te Velde ER, Pearson PL. The variability of female reproductive aging. Hum Reprod Update. 2002;8:141-54.

van Disseldorp J, Lambalk CB, Kwee J, Looman CW, Eijkemans MJ, Fauser BC, Broekmans FJ. Comparison of inter- and intra-cycle variability of anti-Müllerian hormone and antral follicle counts. Hum Reprod. 2010;25:221-7.

van Rooij IA, Broekmans FJ, Scheffer GJ, Looman CW, Habbema JD, de Jong FH, Fauser BJ, Themmen AP, Te Velde ER. Serum antiMüllerian hormone levels best reflect the reproductive decline with age in normal women with proven fertility: a longitudinal study. Fertil Steril. 2005;83:979-87.

van Rooij IA, Broekmans FJ, te Velde ER, Fauser BC, Bancsi LF, de Jong FH, Themmen AP. Serum anti-Müllerian hormone levels: a novel measure of ovarian reserve. Hum Reprod. 2002;17:3065-71.

Weenen C, Laven JS, Von Bergh AR, Cranfield M, Groome NP, Visser JA, Kramer P, Fauser BC, Themmen AP. Anti-Müllerian hormone expression pattern in the human ovary: potential implications for initial and cyclic follicle recruitment. Mol Hum Reprod. 2004;10:77-83.

Weintraub A, Margalioth EJ, Chetrit AB, et al. The dynamics of serum anti-Müllerian hormone levels during controlled ovarian hyperstimulation with $\mathrm{GnRH}$-antagonist short protocol in polycystic ovary syndrome and low responders. Eur J Obstet Gynecol Reprod Biol. 2014;176:163-7. 